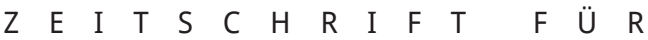

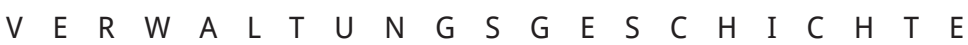

$\begin{array}{lllllllllllll}B & A & N & D & 3 & 2 & 0 & 1 & 8\end{array}$

S E I T

D O I : $10.2478 / \mathrm{ADH}$ I- $2018-0035$

\title{
"Abwanderung und Widerspruch». Zur Aktualität eines analytischen Konzepts des 20. für das 21. Jahrhundert
}

\author{
AGNES ARNDT
}

Als Widerspruch gilt [...] jeder wie immer gearteter Versuch, einen ungünstigen Zustand zu verändern, anstatt ihm auszuweichen, sei es durch individuelle oder kollektive Petition an die unmittelbar Verantwortlichen, durch Berufung an eine höhere Stelle in der Absicht einen Führungswechsel zu erzwingen, oder durch verschiedene Arten von Aktionen und Protesten, einschliesslich jener, die zur Mobilisierung der öffentlichen Meinung dienen sollen. ${ }^{1}$

Mit dieser Arbeitsdefinition führt Albert O. Hirschman ein Begriffspaar in die Sozialwissenschaften ein, dem er in besonderer Weise zutraut, ökonomische ebenso wie nichtökonomische Prozesse zu erhellen. ${ }^{2}$ Präzise gefasst geht es Hirschman um Verfallsprozesse, innerhalb derer das Abweichen von "Normen effizienten, rationalen, gesetzestreuen, tugendhaften oder sonst funktionsgerechten Verhaltens ${ }^{3}$ eklatant wird, im Fall von Unternehmen beobachtbar etwa an einem "Leistungsabfall « ${ }^{4}$ hinsichtlich der Qualität von Waren oder Dienstleistungen. Den Gegensatz zum Widerspruch bildet das Phänomen der Abwanderung, das Hirschman in marktwirtschaftlichen Zusammenhängen entweder als Rückgang der Nachfrage nach einem bestimmten Produkt oder einer Dienstleistung beschreibt oder aber als Austritt aus einem Unternehmen, in politischen Zusammenhängen wiederum als Austritt aus einer bestimmten Organisation, einem Verband oder einer Partei bis hin zum bewussten Verlassen des staatlichen Systems durch Flucht oder Ausreise.

Was Albert O. Hirschmans 1970 erstmals in den Vereinigten Staaten veröffentlichte und 1974 ins Deutsche übertragene Studie "Abwanderung und Widerspruch. Reaktionen auf Leistungsabfall bei Unternehmungen, Organisationen und Staaten« heute wieder aktuell macht, ist die Auseinandersetzung mit einem Idealtypus ökonomischen Verhaltens, das große Teile der Wirtschafts- und Verwaltungswissenschaften als zweckrational definieren und analysieren. ${ }^{5}$ Hirschman dekonstruiert diesen Idealtypus, indem er dem Bild einer rationalen und effizienten, konstant auf ein Leistungsmaximum zuarbeitenden Wirtschaft deren Leistungsschwankungen und -verschlechterungen gegenüberstellt. In Auseinandersetzung mit zeitgenössischen Forschungen, die belegen, dass Unternehmen eben nicht die höchste, sondern in der Regel nur eine befriedigende Gewinnrate erreichen ${ }^{6}$, 
stellt Hirschman die These auf, dass neben einer "straffen «, immer auch die Tendenz zu einer "schlaffen» Wirtschaft bestünde, mehr noch, dass

\section{Schlaffheit nicht nur irgendwie entstanden ist und in bestimmtem Ausmaß in der Welt existiert, sondern daß sie auf Grund einer Art Entropie, die für menschliche Überschussgesellschaften charakteristisch ist, ständig entsteht. ${ }^{7}$}

Dies ist umso bemerkenswerter als das Erscheinen des Buches Anfang der 1970er-Jahre in den USA und in Westeuropa mitten in den Ost-West-Konflikt und die Systemauseinandersetzung zwischen Kapitalismus und Sozialismus fällt. Der Autor bereichert, ohne dies freilich explizit $\mathrm{zu}$ machen, diese Auseinandersetzung um eine erfrischend nüchterne und ideologiefreie Analyse marktwirtschaftlichen Verhaltens.

Abwanderung und Widerspruch bilden nach Hirschman die grundlegenden Mechanismen ab, mit denen der immer wiederkehrenden Tendenz zum Leistungsabfall begegnet werden könnte. Er sei somit, schreibt er 1969 im Vorwort zur amerikanischen Erstausgabe seines Buches, "auf eine Möglichkeit der Analyse bestimmter wirtschaftlicher Vorgänge gestoßen, die einen weiten Bereich sozialer, politischer, ja sogar moralischer Phänomene $\mathrm{zu}$ erhellen ${ }^{8}{ }^{8}$ verspräche. Indem er "Markt- und Nichtmarktkräfte» und somit "wirtschaftliche und politische Mechanismen « als "zwei Hauptakteure mit vollkommen gleichem Rang und vollkommen gleicher Bedeutung « einführe, versuche er, "den Politologen die Nützlichkeit ökonomischer Begriffe und den Ökonomen die Nützlichkeit politischer Begriffe $\mathrm{zu}$ demonstrieren ${ }^{9}$ und die aus seiner Sicht bisher unzureichende Wahrnehmung beider Disziplinen wechselseitig zu befördern. »Vielleicht bedarf es eines Ökonomen, «, so Hirschman,

um unter unseren unterdrückten Kollegen das Selbstgefühl und den Stolz wiederzuerwecken und ihnen das Vertrauen einzuflößen, dass auch ihre Begriffe nicht nur grandeur, sondern auch ein rayonnement aufweisen? ${ }^{10}$

So selbstbewusst dies klingt, so selbstironisch wird es anschließend gewendet. Hirschman bekennt sich $\mathrm{zu}$ der »Besorgnis, die Begriffe >Abwanderung، und ,Widerspruch könnten zu weit gespannt sein«. Diese Sorge habe »in dem Maße« zugenommen, wie sich seine Untersuchung "mit überraschender Leichtigkeit auf immer neue Gebiete ausdehnte«. "Die hauptsächliche Konzession, die ich diesen Sorgen machte, bestand darin«, so der Autor,

dass ich dieses Buch kurz hielt. Im Übrigen aber beschloss ich angesichts der Tatsache, dass ich meine eigene vereinheitlichende Sichtweise für so verschiedenartige Probleme wie den wirtschaftlichen Wettbewerb und das Zweiparteiensystem, die Ehescheidung und den amerikanischen Nationalcharakter, die Black-Power-Bewegung und den Nichtrücktritt hoher staatlicher Funktionäre, die über Vietnam ıunglücklich، waren, gefunden hatte, mich ein wenig gehen zu lassen. ${ }^{11}$

Trotz dieses Bekenntnisses liest sich Hirschmans knapp 100 Seiten umfassende Studie zügig und mit Gewinn, und dies umso mehr, als mit der Finanzkrise der Jahre 2008 und 2009 den Irrationalitäten der Märkte und ihrer Akteure wieder vermehrt Aufmerksamkeit geschenkt wird, mitunter auch unter Verweis auf frühere Ansätze, die zugunsten der Konstruktion eines idealen und vermeintlich rationalen "homo oeconomicus" in den vergangenen Jahrzehnten allzu sehr in den Hintergrund getreten waren. ${ }^{12}$ Neben Kategorien wie Erfolg und Effizienz finden bei Hirschmann auch Emotionen - zum Beispiel das Gefühl der Enttäuschung - Eingang in die Analyse von Unternehmen, Organisationen und Staaten und zwar im Sinne von Ressourcen, die handlungsstiftend wirken können, etwa wenn sie die Bindung an ein Unternehmen fördern oder aber beenden. Die Beobachtung, "that emotions are not only the object of regulation, but also have regulatory potential « ${ }^{13}$, findet hier ihre Entsprechung.

Hirschmans Analyse des Wechselspiels zwischen Marktkräften und außerhalb des Marktes operierenden Kräften beruht auf der Annahme, dass es sich beim Prinzip der Abwanderung um eine wirtschaftliche Logik, beim Prinzip des Widerspruchs dagegen um eine politische Logik handelt. ${ }^{14}$ Abwanderung sei 
charakteristisch für wirtschaftlichen Wettbewerb, der typischerweise als leistungsmaximierend und damit gegenüber Leistungsminderungen als korrigierend erlebt werde. ${ }^{15}$ Es gäbe aber auch Fälle, in denen Wettbewerb effizienzsenkend wirken könne, beispielsweise wenn ein Qualitätsmangel an einem Produkt sich nicht beheben lasse oder wenn Produkte verschiedener miteinander konkurrierender Unternehmen gleichermaßen minderer Qualität seien. In diesen Fällen würde Konkurrenz allenfalls zur unnötigen Abwerbung von Kunden und damit $\mathrm{zu}$ unökonomischer und irreführender Produktdiversifizierung führen. Die Nachteile des Wettbewerbs würden gegenüber möglichen Vorteilen überwiegen. Nach Hirschman würde in solchen Fällen das Ziel der Effizienzsteigerung durch das Mittel des Widerspruchs besser erreicht werden können. ${ }^{16}$

"Aufgabe des Widerspruchs« sei es,

eine Firma oder Organisation auf ihre Fehler aufmerksam zu machen, doch muß dann der Unternehmensführung - der alten oder der neuen - Zeit gegeben werden, auf den Druck, der auf sie ausgeübt wird, zu reagieren. ${ }^{17}$

Dabei steige die Bedeutung des Widerspruchs in dem Maße, in dem sich die Abwanderungsmöglichkeiten verringern. "Jeder Widerspruch" sei somit "vom Standpunkt der Gesundung des Unternehmens bzw. der Organisation per Saldo ein Gewinn «18, zumal da korrekturwirksamer Widerspruch eine qualitätsunelastische Nachfrage - das heißt eine geringe Abwanderung - grundsätzlich ausgleichen könne. Optimal im Sinne einer Maximierung der kombinierten Wirksamkeit von Abwanderung und Widerspruch wäre demnach eine elastische Reaktion der Nachfrage - das heißt eine starke Abwanderung -, dann eine unelastische Reaktion - das heißt eine schwache Abwanderung - und schließlich Widerspruch. ${ }^{19}$ Dieser sei häufig kostspieliger als Abwanderung, er trete eher innerhalb von Organisationen und Unternehmen als außerhalb - etwa im Firmen-Kunden-Verhältnis auf, und seine Erfolgschancen seien auf Märkten mit geringer Kundenanzahl sowie bei teuren Produkten - wie Immobilien und Kraftfahrzeugen - größer als in entwickelten Wirtschaften mit einer Vielzahl von
Märkten und Gütern. ${ }^{20}$ Während die Abwanderung eine Entweder-Oder-Entscheidung ist, sei der Widerspruch eine "Kunst, die sich ständig in neue Richtungen « ${ }^{21}$ entwickle.

Widerspruch in Unternehmen ebenso wie in öffentlichen Verwaltungen werde insbesondere dann wichtig, wenn Abwanderung praktisch ausgeschlossen ist, während umgekehrt eine starke Abwanderung einen wirksamen Widerspruch oft unterdrückt. Daher sei meist festzustellen, dass der eine Mechanismus gegenüber dem anderen dominiere, selten jedoch beide gleichzeitig und gleichgewichtig auftreten. Eine Ausnahme allerdings bildeten politische Parteien und auf Freiwilligkeit beruhende Vereinigungen. Im Rahmen der Untersuchung dieser Organisationstypen führt Hirschman einen weiteren Begriff in die Untersuchung ein, der in der amerikanischen Fassung seiner Studie noch im Titel enthalten ist, in der deutschen Übersetzung jedoch fehlt. Widerspruch wird hier als eine spezifische Spielart der Loyalität beschrieben, deren Funktion es sei, die Abwanderung $\mathrm{zu}$ hemmen und den Widerspruch zu fördern. Das Gefühl der Loyalität steigere einerseits die Bereitschaft, den Gewinn eines besseren Produktes, der durch Abwanderung entstehen würde, preiszugeben und erhöhe andererseits das Interesse, innerhalb einer Organisation Einfluss zu gewinnen.22 Somit könne Loyalität die Tendenz qualitätsbewusster Mitglieder oder Kunden, als erste abzuwandern, neutralisieren und die übermäßige Steigerung von Qualitätsverlust regulieren. "Als Paradoxon formuliert [...] [sei] die Loyalität dann am sinnvollsten [...], wenn sie am irrationalsten aussieht «², so Hirschman.

DieStudie endet mit dreiEmpfehlungen zur Erhaltung der Widerstandskraft gegen den Leistungsabfall: Organisationen und Verwaltungen, die primär auf einen Reaktionsmechanismus eingestellt seien, bräuchten hin und wieder eine "Injektion" des jeweils anderen Mechanismus‘. Andere Organisationen müssten Zyklen durchlaufen, in denen sich die beiden Mechanismen als „jeweils dominant“ abwechseln würden. Schlussendlich sei es aber das Bewusstsein über die natürliche Instabilität einer optimalen Mischung, das zu einem verbesserten Aufbau von Institutionen beitrüge, die sowohl Abwanderung als auch Widerspruch brauchten, um funktionsfähig zu bleiben. Ein wenig überraschend 
nach der eher lakonischen Einleitung endet das Buch mit der seitens des Autors geäußerten Hoffnung auf einen »direkteren Einfluss» seiner Arbeit:

\section{Dadurch, dass [sie] auf das verborgene Wirkungspotential der jeweils gerade vernachlässigten Reaktionsweise aufmerksam macht, könnte [sie] - je nachdem - den Rückgriff auf die Abwanderung oder den Widerspruch fördern. Das ist jedenfalls der Stoff, aus dem Träume von Autoren gemacht sind. ${ }^{24}$}

20 Jahre später veröffentlicht der Ökonom in der Zeitschrift »Leviathan « einen »Essay zur konzeptuellen Geschichte«, der das von ihm eingeführte Begriffsinstrumentarium auf das Ende der Deutschen Demokratischen Republik zu beziehen sucht.. ${ }^{25} \mathrm{Er}$ untersucht den "Antagonismus zwischen privater Abwanderung und öffentlichem Widerspruch", der, seiner Ansicht nach, in der DDR insofern besonders bedeutsam war, als

\section{bereits vom Tag ihrer Gründung an die Stabilität der Deutschen Demokratischen Republik unterminiert [wurde] durch ihre Koexistenz mit der Bundesrepublik. Die Möglichkeit, das eigene persönliche Los zu verbessern, indem man einfach wegging und in das andere, größsere, freiere und dann bald auch wohlhabendere Deutschland umzog, unterscheidet fundamental die ostdeutsche Nachkriegserfahrung von der der Polen, Tschechen und Ungarn [...]. ${ }^{26}$}

Auch wenn er mit diesem Befund den Abwanderungsbewegungen aus den genannten Ländern wohl eine zu marginale Rolle zuschreibt - die politische und soziale Tragweite der freiwilligen, vor allem aber auch unfreiwilligen Emigration gerade auch der intellektuellen Eliten Ostmitteleuropas ist nicht $\mathrm{zu}$ unterschätzen ${ }^{27}$ - hat Hirschman doch insofern Recht, als der Widerspruch gegen die sozialistischen Systeme zwar unter anderem in der DDR mit dem Arbeiteraufstand 1953 seinen Anfang nahm. Die Widerstände in Ungarn 1956, in der Tschechoslowakei 1968 und - durch Gründung der Solidarność - in Polen 1980 unterschieden sich allerdings tatsächlich vom ostdeutschen Beispiel, weil sie - trotz ihrer oft gewaltsamen Unterdrückung im Untergrund weitergeführt wurden und »immer neue Spielräume für die bürgerliche Gesellschaft $\star^{28}$ schufen. Für die DDR hingegen macht Hirschman »bis zur machtvollen Bewegung vom Herbst 1989 « $^{29}$ das Prinzip der Abwanderung als kennzeichnendes Element der Opposition aus. Mehr noch: Er findet Belege dafür, dass »in einem Land zu leben, das ständig von seinen Bürgern aufgegeben werde«, jedenfalls einige der verbleibenden Bürger, ihrem Erleben nach, "physisch und psychisch krank mache ${ }^{30}$

Es ist keine wertende oder gar abwertende Beobachtung, die Hirschman hier mit Bezug auf jene Ostdeutschen macht, die sich vor 1989 gegen den Widerspruch und für die Abwanderung entschieden. Vielmehr betont er, wie sehr die Abwanderung "viele der loyaleren Bürger, auch und gerade jene, die keinerlei Absicht hatten abzuwandern [...] beeindruckte, deprimierte und alarmierte « und schließlich doch zur Artikulation von öffentlichem Widerspruch und der Entstehung der Bürgerbewegung motivierte.

Bei einem gewissen Grad der Besorgnis entschieden sie, sich zu Wort zu melden. Die Mobilisierung und der Widerspruch dieser Bürger kann daher als ein Ersatz für die mangelhafte Reaktion der DDR-Manger, gleichsam als ein zweiter Weckruf gesehen werden. ${ }^{31}$

Und doch gewinnt man bei der Lektüre von Hirschmans Arbeiten zu "Abwanderung und Widerspruch" insbesondere wenn man sein 1972 erschienenes Buch mit seinem 1992 publizierten Aufsatz kontrastiert - den Eindruck, dass der Autor dem Prinzip des Widerspruchs mehr Geltung verschaffen möchte:

Ich wurde mir des Interesses an meinem zwanzig Jahre alten Buch bewusst, als ich das akademische Jahr 1990/91 am Wissenschaftskolleg in Berlin verbrachte. Zu der Zeit war es mir zudem möglich, mich durch Lektüre und Interviews näher mit der Geschichte der DDR und insbesondere mit der bemerkenswerten Geschichte ihres Endes bekannt zu machen. Ich kam zu dem vielleicht wenig überraschenden Schluss, dass die Perspektive, die das Begriffspaar ,Abwanderung und Widerspruch, eröffnet, tatsächlich hilfreich sein könnte, um 
einige der deutschen Ereignisse in einem neuen Licht zu betrachten, und dass diese Begriffe ihrerseits bereichert werden könnten durch das Zusammentreffen mit einem großen historischen Testfall. Um meine Einleitung mit einer persönlichen Note zu beenden: Nach einer Abwesenheit von mehr als einem halben Jahrhundert von dem Land, in dem ich meine ersten achtzehn Jahre verbracht habe, ermöglichte mir dieses Thema einen Wiedereintritt in deutsche Politik und Geschichte. ${ }^{32}$

Albert O. Hirschman, der am 7. April 1915 als OttoAlbert Hirschmann in Berlin geboren wurde, verließs Deutschland 1933, nachdem er als Sohn einer jüdischen Familie und Mitglied der Sozialistischen Arbeiterjugend SAJ an der damaligen Friedrich-Wilhelms-Universität Unter den Linden ein Studium der Nationalökonomie aufgenommen hatte. Er setzte seine akademische Ausbildung zunächst an der Sorbonne fort und konnte sie dort 1935 mit Diplom abschließen, um daraufhin mit einem Stipendium des International Student Service von 1935 bis 1936 an der London School of Economics zu studieren. Nachdem er kurz auf republikanischer Seite im spanischen Bürgerkrieg gekämpft hatte, ging er im Herbst 1936 nach Italien, promovierte 1938 an der Universität Triest mit einer Arbeit über die französische Außenhandelspolitik, musste aber aufgrund der faschistischen Rassengesetze bereits im Herbst 1938 das Land verlassen. Bis zum Juni 1940 gehörte er freiwillig der französischen Armee an und floh schließlich nach deren Kapitulation in die Vereinigten Staaten, wo er in der US Army, zunächst in Nordafrika, dann in Italien, diente. Neben Stationen an führenden Universitäten der USA, unter anderem von 1941 bis 1943 an der University of California, seit 1956 als Gastprofessor an der Yale University und seit 1974 als Professor am Institute for Advanced Study in Princeton, war Hirschman auch an zentralen Schnittstellen zwischen Wirtschaftswissenschaft und Politik tätig: ab 1946 als Ökonom bei der amerikanischen Zentralbank und beim Marshall-Plan und von 1952 bis 1956 als Berater der kolumbianischen Regierung. ${ }^{33}$ Die Freie Universität Berlin, die Hirschman 1988 mit einem Ehrendoktortitel ehrte, sieht in der interdisziplinären Wirkungsweise Hirschmans sowie

\author{
in seinem Leben und Werk die Traditionen des \\ europäischen Humanismus [verwirklicht], in dem trotz \\ aller Einsichten in die Unzulänglichkeiten menschlichen \\ Handelns die Hoffnung auf eine glückliche Zukunft der \\ Menschheit nicht verloren geht. ${ }^{34}$
}

Im Oktober 1972 verfasste Hirschman ein knappes Vorwort zur deutschen Ausgabe seiner Studie. "Nach der Veröffentlichung des Buches in den Vereinigten Staaten " sei er sich dessen bewusst geworden, dass es »tiefere Gründe« gebe, die ihn »an das Thema fesselten«:

\begin{abstract}
Ein großer Teil des Buches kreist um die Besorgnis, dass durch die Abwanderung jener, deren Widerspruch unüberhörbar wäre, ein besonders wirksames Mittel gegen den Niedergang verloren gehen könnte. Es gibt wohl eine Verwandtschaft zwischen dieser Situation und dem Schicksal der Juden, die noch nach 1939 in Deutschland waren. Die meisten Jungen und Tatkräftigen wanderten, wie ich, in den ersten Jahren nach Hitlers Machtergreifung $a b$, und hinterließen eine ernstlich geschwächte Gemeinschaft. Sicher gab es damals praktisch keine Möglichkeit für einen wirksamen Widerspruch, wer immer auch ging oder blieb. Dennoch, der eigentliche Ursprung des Buches mag wohl ein sorgfältig unterdrücktes Schuldgefühl sein, das einfach da ist, wenn es auch verstandesmäßig absurd erscheint. Die deutsche Ausgabe dieses Buches [ermögliche es ihm], diesen Nachgedanken zum Ausdruck zu bringen. ${ }^{35}$
\end{abstract}

Es ist nicht die Betroffenheit über diesen Absatz, die dazu führt, dass man - entgegen aller wissenschaftlichen Gepflogenheiten - Albert O. Hirschmans Buch weniger zeitgenössische Relevanz wünschte. Das von ihm eingeführte Begriffspaar erhellt tatsächlich »einen weiten Bereich sozialer, politischer, ja sogar moralischer Phänomene ${ }^{36}$ und benennt Handlungsoptionen für Wirtschafts- wie Verwaltungszusammenhänge. Überträgt man die vom Autor gewählten empirischen Beispiele in die Gegenwart, drängt sich allerdings die Frage auf, ob nicht die Verfügbarkeit über diese Handlungsmodi als solche stärker ins Zentrum von Analysen ökonomischer und politischer Institutionen rücken sollte. Es ist die Wahlmöglichkeit zwischen Abwanderung und Widerspruch, zwischen Loyalität 


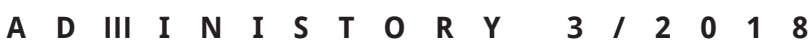

und Enttäuschung, die zu einem Privileg wird, wenn wirtschaftliche, politische und soziale Bedingungen die zur Verfügung stehenden Handlungsmuster mehr und mehr reduzieren. Angesichts von globalen Migrationsbewegungen zu Beginn des 21. Jahrhunderts, die selbst jene des 20. Jahrhunderts in den Schatten stellen, ist Hirschmans Analyse und sein Plädoyer für die korrigierende Kraft der Wechselwirkung von Abwanderung und Widerspruch in Unternehmungen, Organisationen und Staaten aktueller denn je - mit ungewissem Ausgang. 
1 Vgl. Albert O. Hirschman: Abwanderung und Widerspruch. Reaktionen auf Leistungsabfall bei Unternehmungen, Organisationen und Staaten, Tübingen 1974, S. 25. Die Erstausgabe erschien 1970 bei Harvard University Press, vgl. Ders.: Exit, Voice, and Loyalty. Responses to Decline in Firms, Organizations, and States, Cambridge 1970.

2 Zu Hirschmans Werk und Biografie vgl. Jeremy Adelman: Worldly Philosopher. The Odyssey of Albert O. Hirschman, Princeton 2013, zur Rezeptionsgeschichte von „Exit, Voice, and Loyalty" insbesondere S. 440-454.

3 Vgl. Hirschman, Abwanderung und Widerspruch, S. 1.

4 Vgl. ebd., S. 3.

5 Vgl. ebd., S. 1-3.

6 Vgl. ebd., S. 9-10 und Ders.: Die Strategie der wirtschaftlichen Entwicklung, Stuttgart 1967; sowie Herbert A. Simon: »A Behavioral Model of Rational Choices«, in: Quarterly Journal of Economics 69 (1952), S. 98-118; Gary S. Becker: »Irrational Behavior and Economic Theory«, in: Journal of Political Economy 52 (1962), S. 1-13; Harvey Leibenstein: »Allocative Efficiency versus X-Efficiency«, in: American Economic Review 56 (1966), S. 392-415; Richard Cyert/James March: A Behavioral Theory of the Firm, Englewood Cliffs 1963.

7 Vgl. Hirschman: Abwanderung und Widerspruch, S. 12.

8 Vgl. Albert O. Hirschman: "Vorwort zur amerikanischen Ausgabe», in: ders.: Abwanderung und Widerspruch, S. V-VI, hier S. V.

9 Vgl. Hirschman: Abwanderung und Widerspruch, S. 16.

10 Vgl. ebd., S. 16.

11 Vgl. Hirschman: "Vorwort zur amerikanischen Ausgabe«, S. V-VI, hier S. V.

12 Vgl. George A. Akerlof / Robert J. Shiller: Animal Spirits. How Human Psychology Drives the Economy, and Why it Matters for Global Capitalism, Revised edition, Princeton 2010.

13 Vgl. Peter Becker / Peter Collin / Timon de Groot / Robert Garot: "Bureaucracy and Emotions - Perspectives across Disciplines", in: Administory. Journal for the History of Public Administration/ Zeitschrift für Verwaltungsgeschichte 3 (2018), S. 5-19, hier S. 7.

14 Vgl. Hirschman: Abwanderung und Widerspruch, S. 13-15.

15 Vgl. ebd., S. 17-21.

16 Vgl. ebd., S. 21-24.

17 Vgl. ebd., S. 27.

18 Vgl. ebd., S. 27.

19 Vgl. ebd., S. 30-32.

20 Vgl. ebd., S. 30-32.

21 Vgl. ebd., S. 30-32.

22 Vgl. ebd., S. 66-67.

23 Vgl. ebd., S. 66-67.

24 Vgl. ebd., S. 107.

25 Vgl. Albert O. Hirschman: «Abwanderung, Widerspruch und das Schicksal der Deutschen Demokratischen Republik. Ein Essay zur konzeptuellen Geschichte«, in: Leviathan. Berliner Zeitschrift für Sozialwissenschaft 20 (1992), S. 330-358, hier S. 358.

26 Vgl. ebd., S. 335.

27 Vgl. Agnes Arndt: Rote Bürger. Eine Milieu- und Beziehungsgeschichte linker Dissidenz in Polen, Göttingen 2013, S. 167-178.

28 Vgl. Hirschman: „Abwanderung, Widerspruch und das Schicksal der Deutschen Demokratischen Republik«, S. 335..

29 Vgl. ebd., S. 335.

30 Vgl. ebd., S. 352. Hirschman zitiert hier Christoph Hein sowie, im weiteren Verlauf seines Textes, Intellektuelle wie Christa Wolf und Jens Reich.

31 Vgl. ebd., S. 354.

32 Vgl. ebd., S. 332.
33 Vgl. Philipp H. Lepenies: "Possibilism. An Approach to ProblemSolving Derived from the Life and Work of Albert O. Hirschman«, in: Development and Change 39 (2008), S. 437-459, hier S. 441454 sowie Anna Barbara Sum: "Widerspruch als Prinzip, Nachruf auf Albert O. Hirschman (7. April 1915-10. Dezember 2012)«, in: Geschichte und Gesellschaft 39 (2013), S. 125-138.

34 Vgl. Prof. Dr. Albert O. Hirschman, http://www.wiwiss.fu-berlin. de/fachbereich/ehrenpromotionen/hirschman.html, zuletzt eingesehen am 15. August 2018.

35 Vgl. Hirschman: „Vorwort zur deutschen Ausgabe«, S. VII.

36 Vgl. Hirschman: "Vorwort zur amerikanischen Ausgabe», S. V-VI, hier S. V. 


\section{Abstract}

Albert O. Hirschman's Exit, Voice, and Loyalty. Responses to Decline in Firms, Organizations, and States (1970, in German 1974) deconstructs a stereotype of economic behavior that large parts of social sciences define and analyze as rational and goal-oriented. Hirschman confronts the image of a rational and efficient economy, constantly working towards maximizing output, with the fluctuation and deterioration of its performance. Exit and Voice constitute, according to Hirschman, the fundamental mechanisms available to counteract this tendency for performance decline. He introduces market and non-market forces into his analysis, thereby addressing both economic and political mechanisms, in order to advance the mutual recognition of both disciplines, which Hirschman deems insufficient. At the time of its publication, Hirschman's study was appreciated for its cross-disciplinary approach. In the eyes of the author however, what grants the book current relevance is its analytical compatibility with devastating present-day problems.

\section{About the Author}

Agnes Arndt (Max Planck Institute for Human Development) is a historian of modern and contemporary Europe. She was a Gerald D. Feldman Fellow at the German Historical Institutes in London, Paris and Warsaw and has published two monographs on the political transformation in East Central Europe: Rote Bürger: Eine Milieu- und Beziehungsgeschichte linker Dissidenz in Polen (Vandenhoeck \& Ruprecht, 2013) and Intellektuelle in der Opposition: Diskurse zur Zivilgesellschaft in der Volksrepublik Polen (Campus, 2007). She is currently working on a book on the relationship between emotions, entrepreneurship and capitalism in Germany from the 1840 s to the present. 